\title{
Optical techniques for in situ dynamical investigation of plastic damage
}

\author{
Christophe Baravian • Stéphane André • \\ Norbert Renault • Nadjim Moumini $\cdot$ Christian Cunat
}

Received: 30 June 2007 / Accepted: 28 November 2007

(C) Springer-Verlag 2007

\begin{abstract}
We follow the damage process of high-density polyethylene during tensile tests. We simultaneously track changes in the density and average orientation of cavities using incoherent light transport. At the same time, we measure the true strain with a video-extensometer and the heat with an infrared imager. We see that the damage process has two major separate steps. First, a globally isotropic nucleation and growth of cavities occurs up to a deformation of about 1.1. Then, at higher deformations, cavities stop growing. Instead, they progressively orient and elongate along the tensile axis. The transition between these two damage processes seems to be related to strong physical and geometrical constraints, also probed through a typical thermal signature.
\end{abstract}

Keywords Plastic damage - Cavitation .

Videoextensometry $\cdot$ Thermomechanics $\cdot$ Light scattering

\section{Introduction}

Many studies aim to understand the mechanisms occurring in the deformation of semicrystalline polymers (SCP). The complex nature of SCPs makes them interesting materials from a physical point of view. In particular, the presence of both crystalline and amorphous phases has induced various experimental observations of damage phenomena (when

Paper presented at the 4th Annual European Rheology Conference, (AERC), April 12-14, 2007, Naples, Italy.

C. Baravian $(\varangle) \cdot S$. André $\cdot$ N. Renault $\cdot$ N. Moumini $\cdot$ C. Cunat Laboratoire d'Energétique et de Mécanique Théorique et Appliquée, Nancy University,

CNRS UMR 7563. 2, Avenue de la forêt de Haye, BP160,

54504 Vandoeuvre lès Nancy Cedex, France

e-mail: christophe.baravian@ensem.inpl-nancy.fr they are significantly deformed) and a succession of conjectures about their origin, essence, modes of evolution, interactions, etc.

The recent literature (Argon 1997; Hiss et al. 1999) agrees that it is difficult to identify the routes by which the initial structure of an SCP specimen is deformed and its texture profoundly modified. Consequently, there is no pertinent explanatory physical model at the macroscopic level (i.e., a behavior law). Nevertheless, all the static morphological states taken by the microstructure at various stages of the deformation history have been identified. This is due to a constant improvement of the physical techniquesand of their constantly increasing accessibility — so that bulk matter can be studied over length scales ranging from the Angstrom to the millimeter. These techniques are all based on a probe of the structural network according to particle or wave radiation. The morphological elements composing SCPs are well understood, from the scale of the monomer up to the submillimeter scale where a first type of structure (spherulites) is encountered due to organization towards the solid state during the cooling from the liquid phase.

Unlike experimental investigations at the macroscopic level, this knowledge is (unfortunately) more based on qualitative information obtained from image analysis rather than on a physical model. Therefore, the long experience of research teams specialized in the interpretation of the images produced by some particular technique (electron microscopy or X-ray scattering, for instance) is crucial, but acts as a brake upon an appropriation of this knowledge by the whole community. It is also important to mention that most of the techniques used to investigate the material organisation at small scales (nanometer) only probe limited volumes of matter and, therefore, mainly provide local information. Another limit is that these techniques can only be used to study "static states": The sample microstructure 
is examined in a "postmortem" step, i.e., after controlled pre-deformation. This approach is necessary because the instruments needed for electron microscopy, X-ray scattering, nuclear magnetic resonance, microcalorimetry, etc. cannot be integrated into the testing machine. A future challenge is to develop testing machines small enough to allow in situ characterization with these techniques.

This paper focuses on the use of a recently developed light scattering technique, incoherent steady light transport, for microstructural characterization. It avoids the drawbacks mentioned above and has several other advantages.

First, the apparatus is light, portable, and compact. Acquisition times are short, less than a second, so it can be used for real-time in situ sample inspection during a mechanical test.

Second, the technique measures parameters characterizing the microstructure, and these parameters are measured dynamically with respect to the loading paths applied to a representative volume element of the matter. Therefore, it provides quantitative data, and thus, objective information that can be used to check the pertinence of micro- or macromechanical models.

Third, this technique scans 3D volumes of matter of the order of a few cubic millimeters, producing spatially averaged information about the microstructure at the sample scale.

This technique probes the microstructure only for a partial range of length scales depending on the probing source. The technique is described in detail elsewhere (Baravian et al. 2005; Caton et al. 2007; Parker et al. 2007). It relies on incoherent steady light transport (ILT) in the material and typically characterizes objects with sizes between 0.1 and a few hundreds of micrometers. Therefore, it is well adapted to study mechanical damage, as it allows in situ measurements of parameters connected to the development of voids or cavities in the test sample. It can quantify the visible whitening of damaged high-density polyethylene (HDPE).

Cavitation only occurs in tension, so it is often considered as not intrinsic to the material but just a side effect of plastic deformation. Nevertheless, understanding the microstructural mechanisms responsible for this catastrophic localization is also a way to understand the physics of phenomena like the role of crystallographic slips or of the network of entanglements in the amorphous phase.

One disadvantage of the technique is that it can only measure turbid materials. However, there remain a large range of polymers (amorphous or SCP) to which it can be applied. One of the subjects of this paper is a new field of application of the technique. It was initially used to study the rheo-optical behavior of turbid suspensions (Baravian et al. 2004, 2007; Mougel et al. 2006). One should finally mention that another light scattering technique has been developed earlier with the same objective (Schirrer et al. 1997; Géhant and Schirrer 1999). It relies on coherent light transport. The underlying physical principles and basic characteristics of the two techniques are similar. They give access to the photon transport length and, using a model of the interaction of photons with the scatterers, based on classical theories, both techniques give at least one parameter related to the number density of scatterers and their average size. The incoherent steady light transport technique has several advantages over the coherent technique. First, there is no need for calibration. Second, the intensity of the signal is high because most of the detected photons are incoherent in such highly turbid media. Third, it easily detects the presence of anisotropy in the turbid medium. Fourth, the technique is less demanding technically, as there is no need for the high angular resolution required to measure the coherent backscattering cone (Sapienza et al. 2004). Fifth, ILT can be extended to measure matrix-dependent parameters (and not only the voids). This potential is not fully developed here. It depends on the real-time analysis of polarized light (Dillet et al. 2006; Baravian et al. 2007).

The paper is divided in three sections. In the next section, a brief bibliography summarizes knowledge of the microscopic mechanisms of deformation of SCPs during a tensile test. Then, the material and experimental methods used for in situ characterization of damage are exposed. In particular, we describe the principles of the incoherent light scattering technique and the microstructural parameters that it determines. Finally, the experimental results are presented and interpreted in terms of microstructural damage.

\section{Some fundamentals of damage mechanisms in SCPs}

This literature review is a brief outline of the most important ideas concerning the deformation mechanisms in SCP. The initial ideas have been corrected in the last few decades. Some arguments have been opposed to original schemes in the light of additional experimental results. The complexity of the material has been more precisely taken into account in a more integrated way. In contrast, the early analyses were partial, only considering, for example, the crystalline component. Last, if some precise knowledge on the micromechanisms occurring in an experimental loading path can be retrieved from the microstructure analysis at various stages of deformation, prediction of another loading scheme is still a challenge. For example, studies made in plane-strain compression (Pluta et al. 2000) have shown that while a certain number of active phenomena are shared with those found in uniaxial drawing, other phenomena can only be detected in one or other geometrical configuration of solicitation.

Of course, there have been major reviews since the 1970s that address all the above issues in detail (see for instance Meijer and Govaert 2005). In all cases, the objective was to make some classification or sequencing 
of the key stages of deformation occurring in the sample. They are analyzed in relationship to the identifiable stages in the macroscopic stress-strain curves obtained for uniaxial drawing of HDPE.

Starting from the initial material, which has a homogeneous spherulitic structure, a sequence of three, four, or five stages of deformation are generally considered to occur in the microstructure during cold drawing of SCP (Fig. 1).

Peterlin (1971) identifies three stages of deformation: (1) plastic deformation of the spherulitic structure (pre-neck deformation), (2) discontinuous transformation of the spherulitic into a fibrous structure by micronecking (plastic flow initiation in the neck), and (3) plastic deformation of the fiber structure, inducing gradual strain hardening (an increase of the differential plastic modulus). The microstructure at high deformations (more than 1.5) consists of highly oriented fibers organized into microfibrils with diameters of a few hundred Angstroms and lengths of a few tens of microns. Actually, there are three important types of texturing as a result of the plastic deformation: (1) crystallographic texture (axes of the crystalline lamellae), (2) morphological texture (lamellar orientation), and (3) macromolecular texture (alignment in the amorphous phase). These preferential orientations are in the principal axes of macroscopic deformation (Lee et al. 1993).

In a paper from 1995, Brooks et al. (1995) discuss the occurrence of two successive yield points in a tension test on polyethylene (PE), also defining three stages. On a typical stress-strain curve, the first yield point (Y1) marks the onset of a deformation that is not instantaneously recoverable on unloading. Applied strains greater than Y1, but smaller than the strain at the second yield point $\mathrm{Y} 2$, are recoverable, but after a long time. For deformations beyond Y2, a permanent plastic deformation is observed, for which coarse chain slips lead to lamellar fragmentation (Butler et al. 1997).

Later, Butler et al. (1995, 1997, 1998) used simultaneous time-resolved small-angle X-ray scattering (SAXS) and wide-angle X-ray scattering (WAXS) measurements during

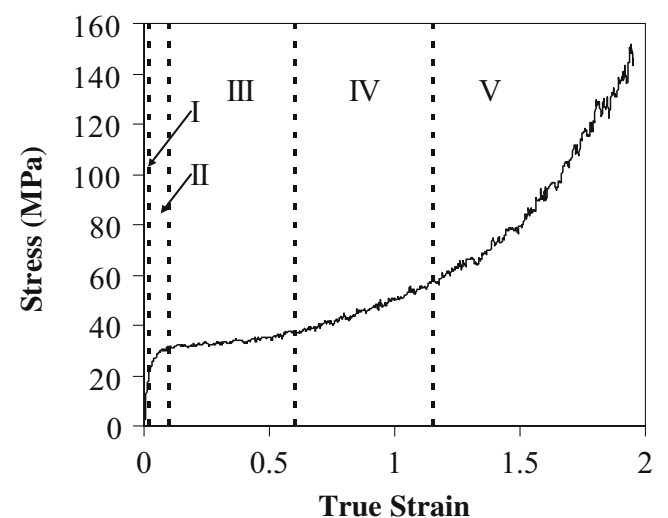

Fig. 1 Experimental true stress-true strain curves for HDPE at a strain rate $\dot{\varepsilon}_{11}=0.005 \mathrm{~s}^{-1}$ cold drawing of various PE to correlate the molecular, lamellar, and macroscopic deformation. They show for various types of HDPE that the first yield point observed on the macroscopic load-extension curve corresponds to (1) the onset of cavitation, coinciding with (2) the onset of the stress-induced martensitic transformation, (3) the onset of crystalline orientation, and (4) a decrease in equatorial long spacing due to lamellar thinning caused by a "fine" chain slip. It also seems that at high strains, during strain softening, a fiber pattern was visible in the SAXS profiles in the "plateau" region (stage III in Fig. 1). The authors refer to an increase in cavitation in this stage, suggesting that microvoids were formed by the release of constraints during lamellar disruption.

In an interesting study, Hiss et al. (1999) reported on uniaxial drawing of PE that associates the macroscopic behavior to observations of the material at various submicrometer scales. The authors consider that deforming an SCP is just the stretching of a network with a high nonlinear viscosity. This idea is motivated by the observation that "drawn samples show an almost complete retraction to their original length when heated up to the melting point". From experiments performed on very different polymers (from solid-like to rubber-like), they derive a common general scheme for the deformation. This scheme relies on the measurement of various recoverable strains: (1) resulting from free shrinkage, (2) resulting from shrinkage due to further heating of the sample at a temperature close to the melting point, and (3) resulting from a constrained shrinkage obtained at zero stress when unloading at the same strain rate as for the loading. In these experiments, the total strain is the sum of the residual or remaining part and of the aforementioned shrunk or recoverable part.

Figure 1 shows their scheme in which the stress-strain curve is divided into five distinct regions by four characteristic points. The first strain range $0 \leq \varepsilon_{11}<0.02$ (stage I) corresponds to the pure Hookean or elastic behavior (no residual strain). From this point, an increase in the compliance is observed (stage II) up to a typical deformation of $\varepsilon_{11} \cong 0.1$ in the vicinity of the yield stress. At stage III $\left(0.1 \leq \varepsilon_{11}<0.6\right)$, a maximum of stress vs the recoverable strain is observed. From this point, a decrease of compliance takes place (stage IV). This transition between stages III and IV $\left(\varepsilon_{11} \cong 0.6\right)$ has also been associated with the appearance of the fibrils in X-ray scattering pattern (or the end of spherulite integrity). Then, a last transition is observed between stages IV and $\mathrm{V}\left(\varepsilon_{11} \cong 1.15\right)$, which generally corresponds to the beginning of the so-called hardening region. Hiss et al. (1999) called it "truly plastic flow" because they noted from this point a truly irreversible and permanent deformation. In stage $\mathrm{V}$, even after prolonged storage at the melting temperature, a residual deformation exists that grows with the total applied strain level. 
At a microstructural level, the evolution from stages I to $\mathrm{V}$ is thought to originate from slip processes (first interlamellar, then intralamellar). It is generally considered that these mechanisms only occur individually in localized zones during stage II before a collective flow regime. Another interesting point is that they consider the transition between stages I and II as the yield point where plastic deformation occurs. They discuss a "nucleation-controlled yield point" that marks the activation of local plastic flows (Salamatina et al. 1994).

Addiego et al. (2006) studied volume variation during tensile tests on HDPE. They consider that in the plastic stage, there is probably competition between a compaction and a dilation effect. Compaction results in a reduction of the average distance between amorphous chains, as shown by X-ray diffraction measurements. The dilation process is explained by a decrease in crystallinity due to a fragmentation process, but also by the formation (nucleation), growth, and coalescence of voids inside and between spherulites. This scheme is based on postmortem scanning electron microscopy (SEM) observations of samples subjected to cold drawing followed by a recovery stage. Results concerning microstructural changes are then analyzed as functions of the residual true axial strain. Existence of coalescence is suggested by SEM. The authors show also that the dilation mechanisms are essentially due to the cavitation process observed in HDPE spherulites. This phenomenon is less active at smaller strain rates. This agrees with the general starting picture of crazing in polymers as stated by Kramer (1983).

\section{Material and experimental techniques}

\section{Material}

The HDPE studied in this work was manufactured by Röchling. Its molecular weight and density are $320,000 \mathrm{~g} / \mathrm{mol}$ and $0.92 \mathrm{~g} / \mathrm{cm}^{3}$, respectively. Differential scanning calorimetry gave a crystallinity index of $66 \mathrm{wt} \%$. The yield stress at $23^{\circ} \mathrm{C}$ and dilation coefficient are given by the manufacturer as 23.44 MPa and 5-6.5 $\times 10^{-5} \mathrm{~K}^{-1}$, respectively. Bone-shaped samples were cut from a 4-mm-thick sheet of polymer and then annealed at a temperature of $120^{\circ} \mathrm{C}$ for $4 \mathrm{~h}$ to produce an isotropic spherulite structure after slow cooling in air.

Video-controlled tensile tests with temperature field monitoring

Mechanical tests were performed on a servo-hydraulic MTS 810 load frame. A video-extensometer (VideoTraction ${ }^{\circledR}$ system, see G'sell and Hiver 1992) allows the live measurement of true strains in the three axes so that the volume strain can be monitored during the test. Another important feature of the system is that it controls the servovalve of the machine in real time so that a regulated true strain rate $\dot{\varepsilon}_{11}$ can be imposed.

Seven dot markers are printed on the front face of the sample. Five of these dots are aligned and equally spaced in the tensile direction for a precise measurement of the true strain in the tensile direction $\varepsilon_{11}$. Two other dots are aligned with the central dot along the transverse direction. They define a representative elementary frame of about $9 \mathrm{~mm}^{2}$. A CCD camera, mounted on a telescopic drive, records images during the test and follows the elementary frame during deformation. PC-based image analysis (Addiego et al. 2006) computes the strains in real time and, hence, changes in volume and cross-section. The computer also records the applied force measured by a load cell so that the true stress can be determined, as the actual cross-section is known. Software now commercialized by Apollor-Union $\mathrm{SA}$, gives the true stress-true strain curve in real time during the test (Fig. 1).

A second optical system consists of an infrared imager (Jade III from CEDIP) mounted on another telescopic drive slaved to the first and placed on the rear face of the sample. It measures the temperature field of the specimen during the test. A mathematical image treatment (not explained here) reconstructs the thermomechanical heat sources as a function of the applied strain. This additional information about thermal effects is rich in information regarding micromechanisms, as we show in "Results and discussion".

A third optical device is associated to these two. It is composed of a laser diode and a CCD camera. These two elements are all that is needed for the incoherent steady light transport technique for in situ characterization of damage by light scattering.

\section{Incoherent light transport experiments}

An incoherent light transport experiment is relatively simple to perform. It consists of a light source (here a laser diode of wavelength $\lambda=635 \mathrm{~nm}$, spot diameter $100 \mu \mathrm{m}$ ) focused on the surface of a turbid medium (the HDPE). The camera (Adimec, 12 bits, $1,024^{2}$ pixels) collects the halo of backscattered light through $4-\mathrm{mm}^{2}$ images centered on the incident light spot. Most of the photons collected far from the laser spot are incoherent because they have been scattered many times before leaving the medium in the backward direction. Figure 2 shows a typical sequences of images acquired during a test. They are treated in two stages.

Spatial intensity decrease

First, we determine the decrease in spatial intensity of the scattered light. For all angles, we average the intensity as a function of radial distance from the laser impact (Fig. 3a). 


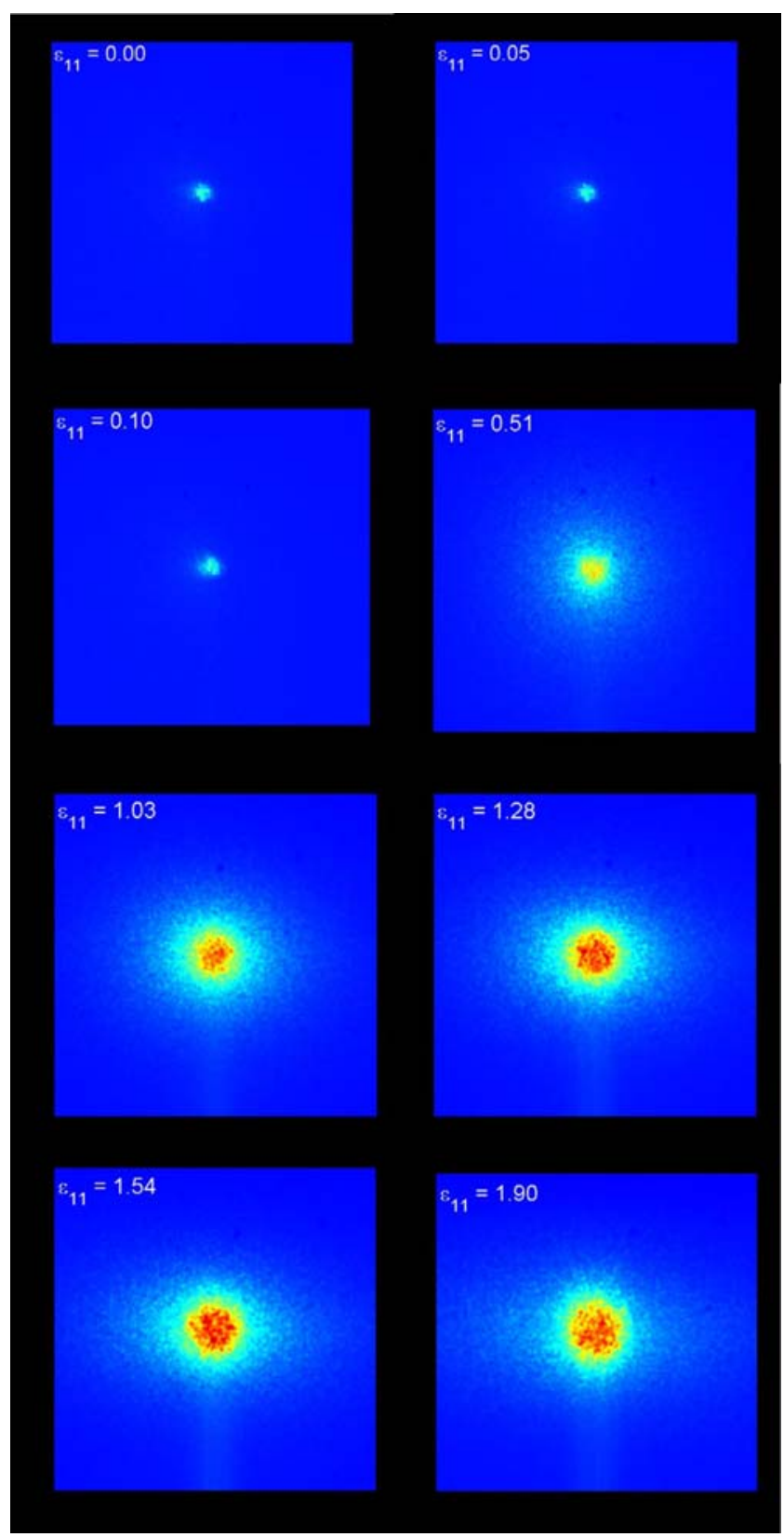

Fig. 2 (color online) Sequence of acquired images at different strains $\varepsilon_{11}$. Strain rate of $0.005 \mathrm{~s}^{-1}$. False color representation in logarithmic scale of intensity

This curve (Fig. 3b) can be well modeled by radiative models in the diffusion approximation (Haskell et al. 1994; Baravian et al. 2005; Caton et al. 2007). Its shape mainly depends on the mean free path of photons inside the medium, $\ell_{T R}$.

Because of the semicrystalline structure of the HDPE, the undamaged sample is already turbid. We find that the corresponding transport length is $\ell_{\mathrm{TR}}^{\text {matrix }}=0.85 \pm 0.1 \mathrm{~mm}$ for all the samples studied. Figure $3 \mathrm{c}$ shows that as the strain increases, the appearance and growth of cavities causes a decrease in the transport length. This also corresponds to an increase in the sample turbidity (a transparent medium has an infinite transport length). The presence of cavities and the undamaged sample turbidity both contribute to the "whiteness" of the sample. The total scattered energy is the sum of the energy scattered by the matrix and the energy scattered by cavities, leading to:

$\frac{1}{\ell_{\mathrm{TR}}}=\frac{1}{\ell_{\mathrm{TR}}^{\text {damage }}}+\frac{1}{\ell_{\mathrm{TR}}^{\text {matrix }}}$

The transport length can be related to microscopic properties of the turbid medium (Baravian et al. 2005; Caton et al. 2007) through three dimensionless parameters: $m=N_{s} / N_{m}$, the refractive index ratio of the scattering object $\mathrm{N}_{\mathrm{s}}$ (1 for cavities) and the surrounding medium $\mathrm{N}_{\mathrm{m}}$ (1.52 for HDPE); the dimensionless size: $x=2 \pi R / \lambda^{\prime}$, where $R$ is the volume averaged scatterers radius and $\lambda^{\prime}$ the wavelength in the surrounding medium; and $\phi$, the volume fraction of scatterers. Knowing $m, x$, and $\phi$, the transport length can be calculated and the incoherent light transport fully modeled (see Caton et al. 2007 for a detailed discussion). When particles are typically larger than a few times the wavelength, the transport length becomes proportional to $R / \phi$, and the proportionality factor (here between $\ell_{T R}^{\text {damage }}$ and $R / \phi$ ) only depends on $m$. In the present case, as we consider that the whitening is only due to the presence of cavities, we use $m \cong 0.66$. The proportionality factor can then be calculated using Mie theory. It is found to be close to 3.14 (Fig. 3d) in the range $0.5 \mu \mathrm{m} \leq R<\infty$. The determination of the transport length $\ell_{T R}$ obtained from image analysis can then be transformed in terms of the damage structure parameter $R / \phi$ using Eqs. 1 and 2 .

$\frac{\phi}{R} \cong 3.14\left(\frac{1}{\ell_{\mathrm{TR}}}-\frac{1}{\ell_{\mathrm{TR}}^{\text {matrix }}}\right)$

\section{Anisotropy}

When the backscattered spot is anisotropic, the second image treatment is used to analyze the angular variation of the backscattered intensity at different radial positions from the laser spot. Incoherent light propagation in anisotropic turbid media has already been used experimentally in other systems (Baravian et al. 2004, Kienle et al. 2004) and studied theoretically by Heino et al. (2003) and Kienle and Hibst (2006). At a given radial distance, we calculate the vertical $\operatorname{Iv}(r)$ and horizontal $\operatorname{Ih}(r)$ intensities as defined in Fig. 4a. The anisotropy is then calculated using $\frac{\mathrm{Iv}-\mathrm{Ih}}{\mathrm{Iv}+\mathrm{Ih}}$. Figure $4 \mathrm{~b}$ shows typical radial variation of the anisotropy. Positive values correspond to image deformation in the vertical direction and negative values to deformation in the horizontal direction, which is also the tensile axis. Images, therefore, possess a double anisotropy, visible in Fig. 2 for 
Fig. 3 (color online) Principles for transport length measurement. a Image and angular integration. b Spatial intensity decrease of light (from a) and adjustment of the diffusion model. The dotted line shows the size of the incident laser spot. c Changes in inverse transport length during a tensile test at a strain rate of $0.005 \mathrm{~s}^{-1}$. d Mie calculation $(m=0.66)$ for the relationship between the transport length and the ratio of cavity radius to volume fraction
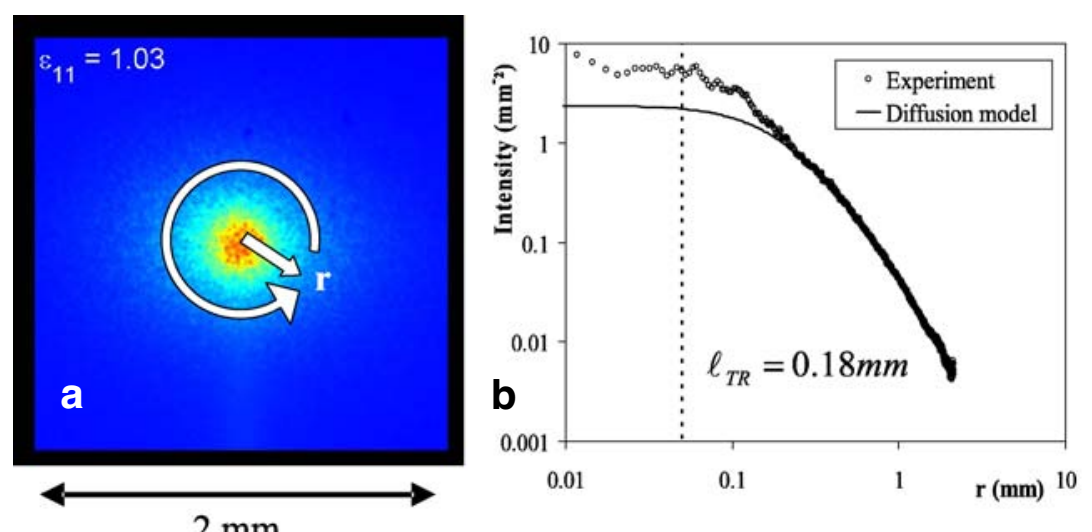

$2 \mathrm{~mm}$
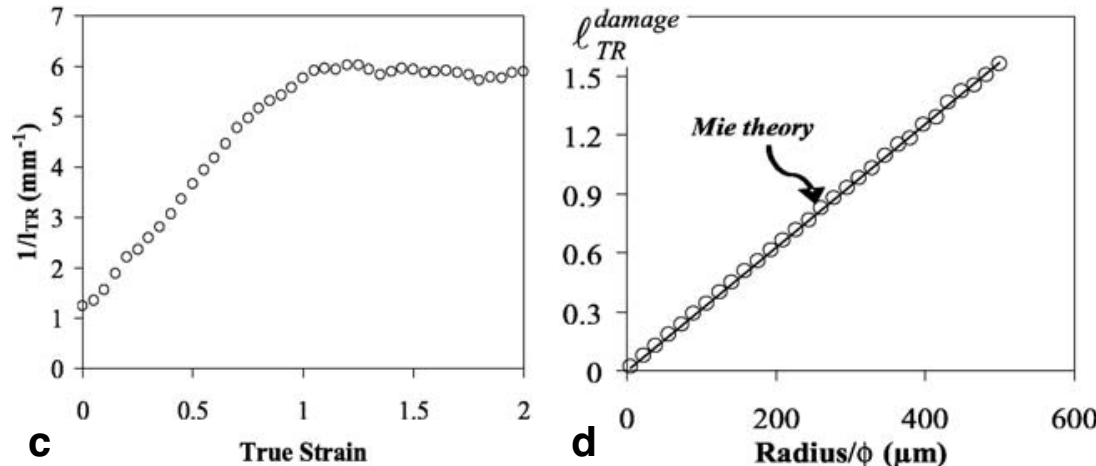

the strain value $\varepsilon_{11}$. The anisotropy close to the center of the image is perpendicular to the anisotropic particles orientation and is parallel to it far from the center of the image (Kienle et al. 2004). Figure 5b shows the anisotropy index calculated as the maximum value of anisotropy in the vertical direction, corresponding to the central anisotropy of images (see Fig. 4b). Other choices have been made for the calculation of the anisotropy index giving similar results.

\section{Results and discussion}

In this section, we describe the experimental results, discuss these observations in the context of the different stages described in the second section, and finally give some physical interpretation of the structural changes occurring in the material.

\section{Thermomechanical response}

In Fig. 5a, the upper curve is the true stress vs dimensionless time. It shows the typical three-stage behavior of HDPE: At small strains, there is a large, quasi-elastic increase in stress (stages I and II), then there is a plateau around a yield stress in the range $30-40 \mathrm{MPa}$ corresponding to some flowing or plastic regime of deformation (stages III and IV), and finally a hardening phase (stage V). After a deformation of 1.87 , the strain is maintained constant.
During this relaxation, the stress decreases from 120 to $60 \mathrm{MPa}$.

Figure 5a also shows the experimental thermomechanical signal extracted by applying our inversion algorithm to

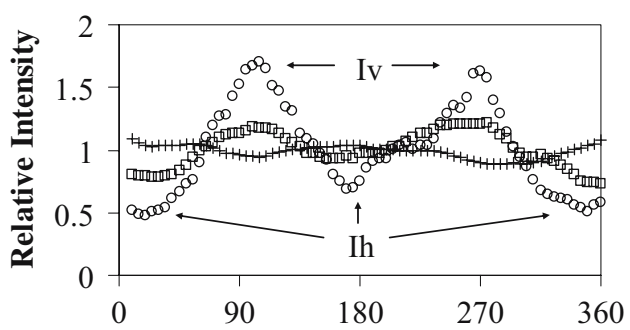

a Angle (degree)

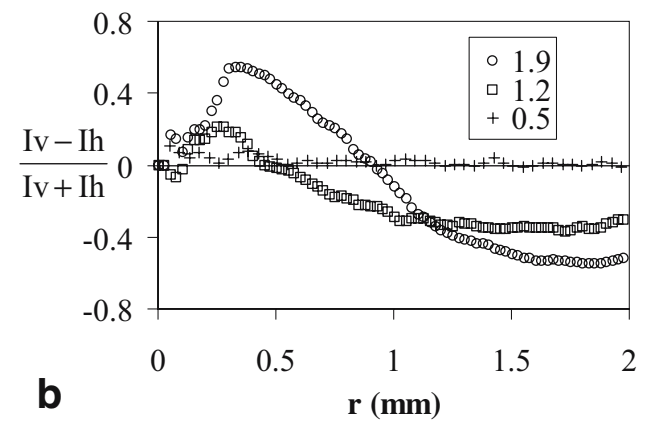

Fig. 4 Incoherent light transport in anisotropic media. Image analysis at three different strains for a strain rate of $0.02 \mathrm{~s}^{-1}$. a Angular variation of relative intensity at a radial position of $0.3 \mathrm{~mm}$. b Radial variation of anisotropy 


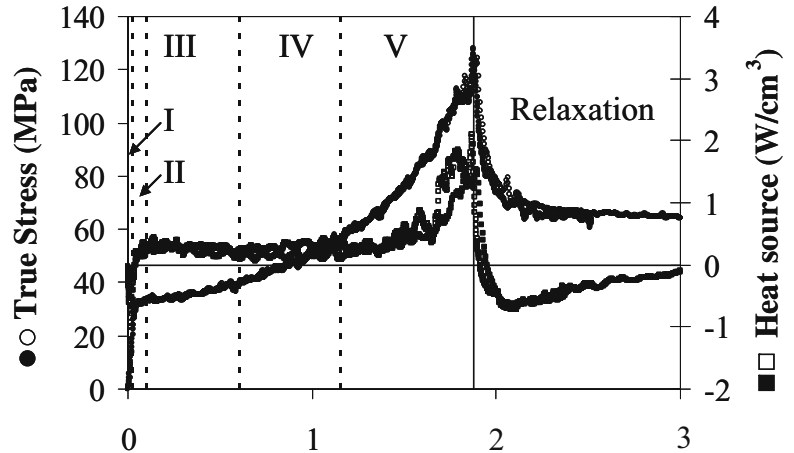

a

Time $\times$ Strain rate

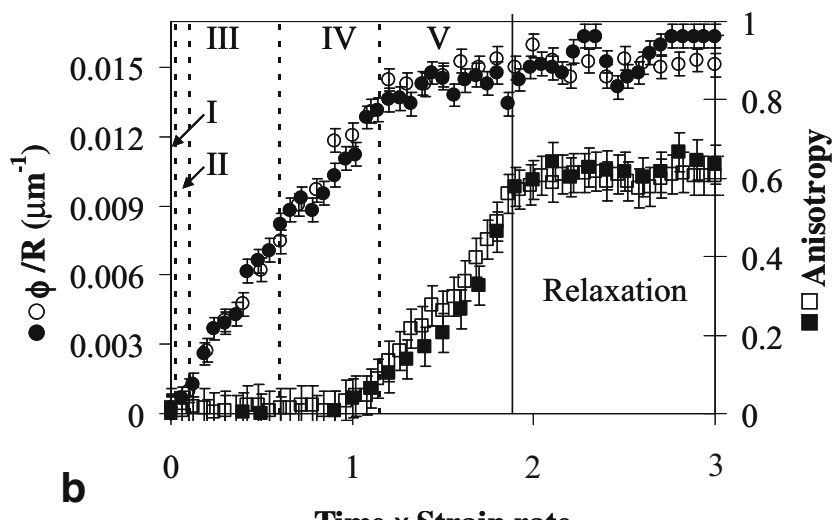

Time $x$ Strain rate

Fig. 5 Tensile test at a strain rate of $0.02 \mathrm{~s}^{-1}$ and reproducibility. a Thermomechanical responses. b Incoherent light transport. After a strain of 1.87 is reached, a relaxation test is performed

a sequence of thermographic images. The ill-posed nature of the inversion problem makes the thermomechanical curves significantly noisier than the signal directly measured from the video-extensometer. Nevertheless, the reproducibility shown in Fig. 5a is satisfactory. This signal can be analyzed to determine the thermal processes accompanying deformation. Four distinct types of behavior can be observed. At early stages (quasi instantaneously), the heat power is negative due to an endothermic reversible process: the well-known thermoelastic coupling (stage I). This effect is clearer in the insert of Fig. 6b. The magnitude of this power decrease corresponds to a thermoelastic coefficient of $16 \times 10^{-5} \mathrm{~K}^{-1}$, which is slightly larger than expected for HDPE. Then, mechanical processes become exothermic. The heat power rapidly rises to a strong positive value (stage II) and then remains constant, characterizing the plastic-flow regime corresponding to stages III and IV. The initiation of the hardening phase (stage $\mathrm{V}$ ) is clearly marked by a strong increase in the heat dissipated, revealing additional processes occurring in the material. During relaxation, a surprising phenomenon occurs: There is a strong endothermic response. A possible explanation is that heat is removed from the surroundings by the HDPE bulk to allow relaxation. In other words, relaxation, considered as a return to an equilibrium state, is possible through some withholding of energy to give new possible pathways for reorganization. This interpretation is being checked by using simulations based on a thermodynamic model for the behavior law of such materials (André et al. 2003).

Incoherent light transport response

The sample visibly changes color during the experiment. Starting from a pale white, the region where high deformation occurs turns to intense white. During the tensile test, the transport length typically falls from 0.85 to $0.15 \mathrm{~mm}$ (Figs. $3 \mathrm{c}$ and $5 \mathrm{~b}$ ): a decrease by nearly a factor of 5 due to development of cavities.

Figure $5 \mathrm{~b}$ shows the changes in the ILT parameters as a function of deformation for the same tensile test as in Fig. 5a. We observe a linear increase of the cavity volume fraction to radius ratio $\phi / R$ during stages III and IV. Then, $\phi / R$ remains approximately constant until the end of the experiment (stage $\mathrm{V}$ and Relaxation). The first images acquired below a strain of 0.1 (the first two images in Fig. 2) show no noticeable change in the light transport (this was further confirmed by additional experiments focusing on this small deformation range). Therefore, stages I and II can also be identified, although ILT cannot be used to distinguish between these two early stages.

Figure $5 \mathrm{~b}$ also shows the anisotropy index measured by ILT as a function of strain. The anisotropy remains very small until a strain value of about 1 (stage I to IV). For deformations above 1, anisotropy appears and increases until the end of the applied strain rate (stage V). No modification of image anisotropy is measurable during relaxation.

Identification of stages in the deformation

We summarize the thermomechanical and light transport observations:

Stage I: This stage is clearly identified by the endothermic response of the material due to the reversible thermoelastic effect (Fig. 5a and insert of Fig. 6b). This criterion unambiguously defines the limit of the elastic domain. Its definition is not obvious from the stress-strain curve (Fig. 5a and insert of Fig. 6a). Elastic deformation in SCPs is generally considered as dominated by deformation of the amorphous component (Butler et al. 1998). The elasticity limit is generally defined through an "immediate" recovery on unloading. This less objective criterion explains that it is generally 
Fig. 6 Effect of strain rate on thermomechanical $(\mathbf{a}, \mathbf{b})$ and incoherent light transport (c, d) responses
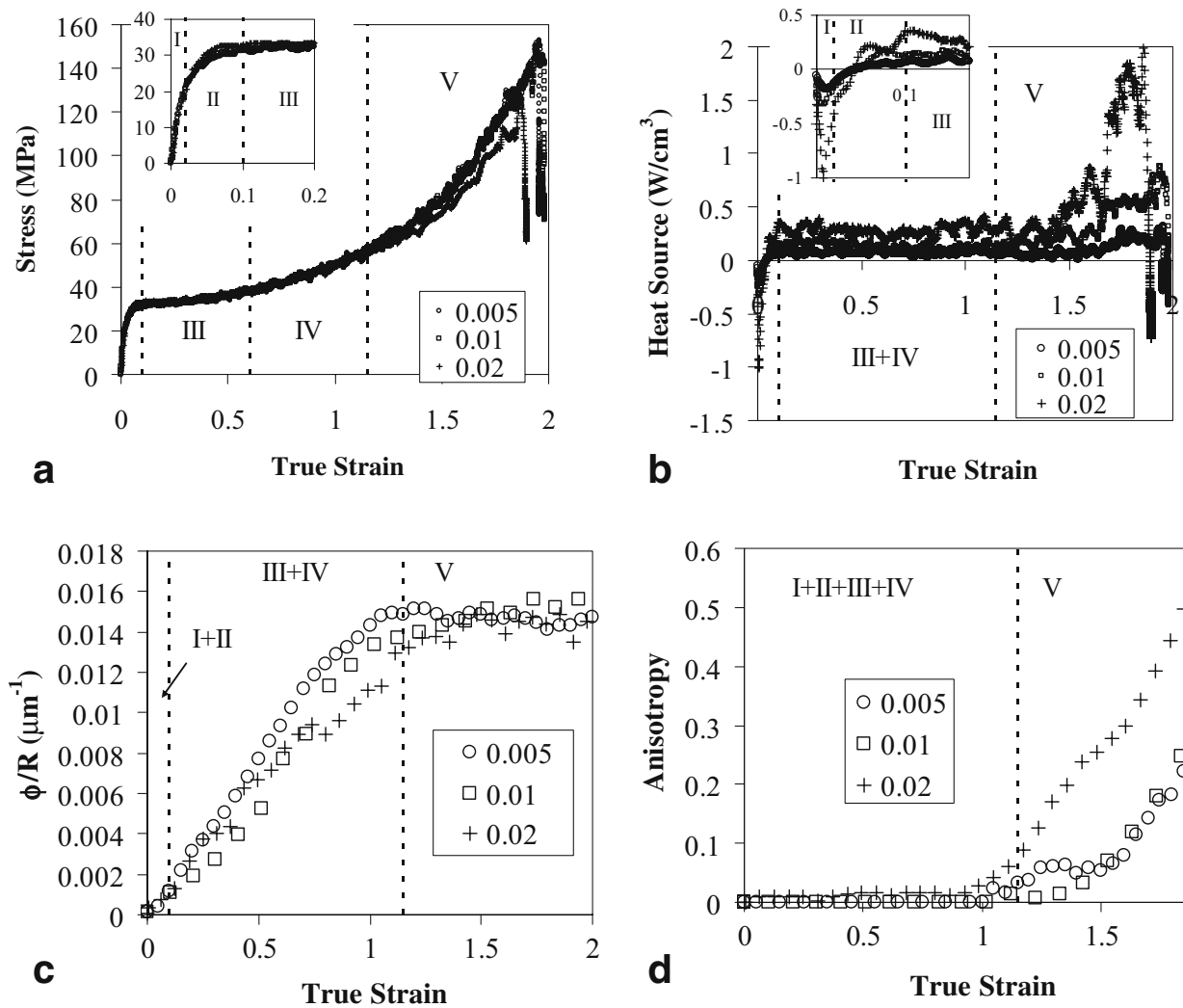

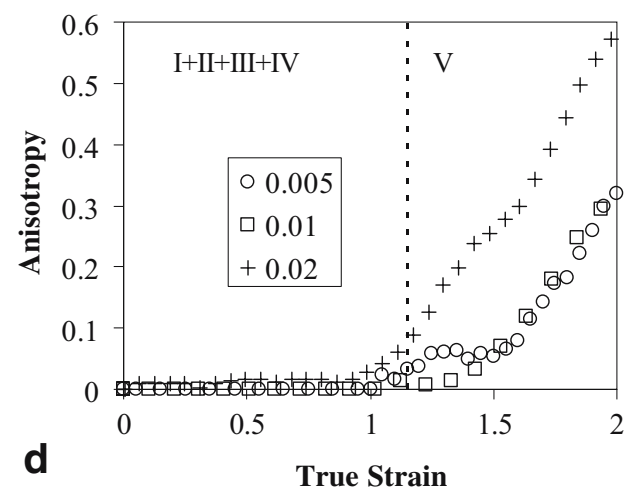

found at higher deformation levels (around 5\% of the applied strain, Brooks et al. 1995).

Stage II: In this stage, the yield stress is reached and, at the same time, the heat source becomes positive (see inserts of Fig. 6a and b). The first active and irreversible thermal effect may correspond to the onset of a stress-induced martensitic transformation, as detected by WAXS measurements in this strain range (Butler et al. 1995) and to the initiation and stabilization of "bubbles" with a radius of $5 \mathrm{~nm}$ and larger (Pawlak 2007). A recent work based on atomic force microscopy also shows that for SCPs, a fragmentation of crystalline lamellae and fibrillation occurs much before the plastic flow (Thomas et al. 2007). Necking of the specimen takes place at the end of this stage, and a first detectable change in the transport length is measured. This transition point between stages II and III corresponds to point Y1 in the paper of Brooks et al. (1995) and point B in Hiss et al. (1999).

Stage III and IV: The heat source remains positive and constant (Fig. 5a). This may indicate that the same physical process occurs during stages III and IV, at least from a macroscopic point of view. Note that in the literature (Butler et al. 1995), both stages are also associated with a continuous morphological transformation from the initial unoriented lamellar state given by the spherulitic structure to the final oriented fibrilar one (Thomas et al. 2007). According to several identified mechanisms of deformation at the microscopic level (fine and coarse chain slips, slip systems, transverse slips, deformation-induced twinning, etc.), the observed phenomenon at the macroscopic level appears as an average collective behavior. These stages therefore correspond to a global plastic flow regime, which, again, is not obvious by just looking at the stress-deformation curve (Fig. 5a). These stages also correspond to the first damage process accessible by the ILT technique, i.e., a decrease of the transport length. This decrease corresponds to a linear increase of $\phi / R$ and no noticeable anisotropy. Thus, the nucleation and growth of cavities is mainly an isotropic process at the sample scale. However, note that this does not necessarily mean that cavities are isotropic. In fact, if cavities are anisotropic but randomly oriented, the incoherent light transport will conserve its polar symmetry (see Baravian et al. 2007 for a detailed discussion). Gaucher-Miri et al. (1996) have noticed a quite clear volume 
increase beyond the yield point as a result of the void initiation during the fibrilar transformation of the spherulitic material, in agreement with ILT results.

Stage V: After a deformation of approximately 1.1, we observe that $\phi / R$ remains constant (Fig. 5b). This means that both the number and volume average size of the cavities remains constant. Appearance of the increasing anisotropy with strain therefore means that either cavities are progressively deformed along the tensile axis (and) or that if they were already anisotropically shaped, the strain increase induces an orientation of them along the tensile axis. We know from our previous studies on anisotropic turbid media that an anisotropy of 0.6 , achieved at a strain rate of $0.02 \mathrm{~s}^{-1}$ and a strain of 2 , corresponds to very elongated and well-oriented scatterers (Baravian et al. 2007). This confirms all experimental studies based on X-ray diffraction that indicate a strong fibrilar microstructure at such strain levels and a fragmentation of crystalline lamellae (Butler et al. 1995, 1997). The initiation of this stage (Point D in Hiss et al. 1999) marks the onset of a true irreversible deformation, assigned to the resorption of entanglements. The network topology changes and produces a loss of memory.

\section{Influence of strain rate}

Figure 6 presents experimental results obtained at strain rates of $0.005,0.01$, and $0.02 \mathrm{~s}^{-1}$. The mechanical responses (Fig. 6a) and the cavity growth (Fig. 6c) seem unaffected by the strain rate in this range. However, Fig. $6 \mathrm{~b}$ shows that the thermomechanical response is affected. Nevertheless, similar stages are observed for all strain rates. More remarkably, at a deformation of 2 , the anisotropy at a strain rate of $0.02 \mathrm{~s}^{-1}$ is nearly twice the anisotropy attained at lower strain rates (Fig. 6d). This may indicate that an unsteady state in cavity development is reached at this strain rate. To our knowledge, this phenomenon has not been observed in previous studies. Despite this much more significant elongation/orientation of cavities, their average size and volume fraction seems to be identical (Fig. 6c). This shows that the change in the deformation mechanism of the HDPE from isotropic growth of cavities to their elongation/orientation is due to a strong physical constraint on cavity growth. This is further confirmed by a systematic increase of the heat source when anisotropy appears, indicating that creating anisotropy has an additional energy cost.

\section{Concluding remarks}

Three non-invasive optical techniques are used to characterize the deformation of HDPE. The video-extensometer gives the behavior law of the material (true stress-true strain relationship) at constant applied true strain rates. The infrared imager gives access to the thermal sources inside the test sample. The incoherent light transport technique characterizes the microstructural development of cavities during the tensile test, averaged over a few cubic millimeters. The coupling of these techniques in real time gives highly complementary information. Used together, all the stages occurring dynamically at different strains can be discriminated. The thermal signal identifies the purely elastic regime (stage I) and the first damage process (stage II), identified by the yield limit in Fig. 6a. Then, a secondary damage process occurs corresponding to nucleation and growth of cavities. The incoherent light transport technique shows that this mechanism is mainly isotropic at the macroscopic level (stages III and IV). This stage corresponds to a plastic flow regime of the material, and the heat source remains constant. Then, a global anisotropy appears in the material and the heat source increases, showing that this third damage process requires more energy (stage $\mathrm{V}$ ).

Acknowledgment We acknowledge Apollor-Union SA (www.apol lor.com) for technical support of this work concerning videoextensometry. We thank Alan Parker from Firmenich SA for useful comments and suggestions.

\section{References}

Addiego F, Dahoun A, G'sell C, Hiver JM (2006) Characterization of volume strain at large deformation under uniaxial tension in highdensity polyethylene. Polymer 47:4387-4399

André S, Meshaka Y, Cunat C (2003) Rheological constitutive equation of solids: a link between models based on irreversible thermodynamics and on fractional order derivative equations. Rheol Acta 42:500-515

Argon AS (1997) Morphological mechanisms and kinetics of large strain plastic deformation and evolution of texture in semicrystalline polymers. J Comput Aided Mater Des 4:75-98

Baravian C, Caton F, Dillet J (2004) Steady light transport application to rheology: a new tool for the characterization of concentrated suspensions. Rheol Acta 43:427-432

Baravian C, Caton F, Dillet J, Mougel J (2005) Steady light transport under flow: characterisation of evolving dense random media. Phys Rev E 71:066603.1-066603.6

Baravian C, Caton F, Dillet J, Toussaint G, Flaud P (2007) Incoherent light transport in an anisotropic random medium: a probe of human erythrocyte aggregation and deformation. Phys Rev E 76:011409

Baravian C, Dillet J, Decruppe J-P (2007) Birefringence determination in opaque media. Phys Rev E 75:032501 
Baravian C, Mougel J, Durand A, Caton F (2007) Characterization of dynamical emulsification process in concentrated conditions. AIChE 53:1994-2000

Brooks NWJ, Duckett RA, Ward IM (1995) Modeling of double yield points in polyethylene: temperature and strain-rate dependence. $\mathrm{J}$ Rheol 39(2):425-436

Butler MF, Donald AM, Brass W, Mant GR, Derbyshire GE, Ryan AJ (1995) A real-time simultaneous small- and wide-angle X-ray scattering study of in-situ deformation of isotropic polyethylene. Macromolecules 28(19):6383-6393

Butler MF, Donald AM, Ryan AJ (1997) Time resolved simultaneous small- and wide-angle X-ray scattering during polyethylene deformation-I Cold drawing of ethylene- $\alpha$-olefin copolymers. Polymer 38(22):5521-5538

Butler MF, Donald AM, Ryan AJ (1998) Time resolved simultaneous small- and wide-angle X-ray scattering during polyethylene deformation-II Cold drawing of linear polyethylene. Polymer 39(1):39-52

Caton F, Baravian C, Mougel J (2007) The influence of the microscopic characteristics of a random medium on incoherent light transport. Optics Express 15(6):2847-2872

Dillet J, Baravian C, Caton F, Parker A (2006) Size determination by use of two-dimensional Mueller matrices backscattered by optically thick random media. Appl Opt 45(19):4669-4678

G'sell C, Hiver JM (1992) Dispositif de caractérisation optique du comportement mécanique local d'une structure pouvant présenter des déformations finies non homogènes. French Patent $N^{\circ} 2823849$

Gaucher-Miri V, François P, Séguéla R (1996) On the mechanisms of initiation and propagation of plastic instability in polyethylene under tensile drawing. J Polym Sci B Polym Phys 34:1113-1125

Géhant S, Schirrer R (1999) Multiple light scattering and cavitation in two-phase tough polymers. J Polym Sci B Polym Phys 37:113-126

Haskell R, Svaasand L, TSay T, Feng T, McAdams S (1994) Boundary-conditions for the diffusion equation in radiativetransfert. J Opt Soc Am A 11:2727

Heino J, Arridge S, Sikora J, Somersalo E (2003) Anisotropic effects in highly scattering media. Phys Rev E 68:031908

Hiss R, Hobeika S, Lynn C, Strobl G (1999) Network stretching, slip processes, and fragmentation of crystallites during uniaxial drawing of polyethylene and related copolymers. A comparative study. Macromolecules 32:4390-4403

Kienle A, Hibst R (2006) Light guiding in biological tissue due to scattering. Phys Rev Lett 97:018104

Kienle A, Forster FK, Hibst R (2004) Anisotropy of light propagation in biological tissue. Opt Lett 29:2617-2619

Kramer EJ (1983) Microscopic and molecular fundamentals of crazing in polymers. In: Kausch HH (ed) Advances in polymer science, 52/53. Springer, Berlin, pp 1-55

Lee BJ, Argon AS, Parks DM, Ahzi S, Bartczak Z (1993) Simulation of large strain plastic deformation and texture evolution in high density polyethylene. Polymer 34:3555-3575

Meijer HEH, Govaert LE (2005) Mechanical performance of polymer systems: the relation between structure and properties. Prog Polym Sci 30:915-938

Mougel J, Alvarez O, Baravian C, Caton F, Marchal P, Stebe M-J, Choplin L (2006) Aging of an unstable w/o gel-emulsion with a non-ionic surfactant. Rheol Acta 45(5):455-560

Parker A, Baravian C, Caton F, Dillet J, Mougel J (2007) Fast optical sizing without dilution. Food Hydrocolloids 21(5-6):831-837

Pawlak A (2007) Cavitation during tensile deformation of highdensity polyethylene. Polymer 48:1397-1409

Peterlin A (1971) Molecular model of drawing polyethylene and polypropylene. J Matter Sci 6:490-508

Pluta M, Bartczak Z, Galseki A (2000) Changes in the morphology and orientation of bulk spherulitic polypropylene due to planestrain compression. Polymer 41:2271-2288

Salamatina OB, Höhne GWH, Rudnev SN, Oleinik EF (1994) Work, heat and stored energy in compressive plastic deformation of glassy polymers. Thermochim Acta 247(1):1-18

Sapienza R, Mujumdar S, Cheung C, Yodh AG, Wiersma D (2004) Anisotropic weak localization of light. Phys Rev Lett 92: 033903

Schirrer R, Lenke R, Boudouaz J (1997) Study of mechanical damage in rubber-toughened poly(methyl methacrylate) by single and multiple scattering of light. Polym Eng Sci 37(10):1748-1760

Thomas C, Ferreiro V, Coulon G, Seguela R (2007) In situ AFM investigation of crazing in polybutene spherulites under tensile drawing. Polymer 48(20):6041-6048 